\title{
Aplicación de la Lógica Difusa en el Control de un Proceso de Prototipo de Pintado
}

\author{
Elvira Rivera-Mujica, $\operatorname{Ing}^{1}$, Pedro Huamaní-Navarrete, Dr $^{2}$ \\ ${ }^{1}$ Universidad Ricardo Palma, Perú, eriveramujica@gmail.com \\ ${ }^{2}$ Universidad Ricardo Palma, Perú, phuamani@urp.edu.pe
}

\begin{abstract}
Este trabajo fue una investigación para un proyecto de tesis en la Carrera de Ingeniería Electrónica, de la Universidad Ricardo Plama. Fue entonces, que nació la idea de implementar físicmanete un prototipo de supervisión y control autónomo de pintado, haciendo uso de un algoritmo de inteligencia artificial principalmente el algoritmo de lógica difusa. Para ello, fue se estableció el uso de dos variables lingüísticas de entrada y una de salida. Asimismo, para la etapa de captura de imágenes, se utilizó una cámara Webcam. Además, se utilizó una PC donde se programó algorítmicamente el Controlador Difuso utilizando el software de computación gráfica LabVIEW. Es así, que como parte del procesamiento, la imagen capturada en el modelo de color RGB fue convertida al modelo de color CYMK, y para ello el algoritmo de Lógica Difusa determinó la cantidad de color requerida salvo en el caso que hubiera ocurrido un error, sea por falta de color o el exceso de este, se tuvo que utilizer la segunda variable lingüística de entrada del controlador difuso. Los resultados obtenidos en este trabajo fueron en que la captura de imagen, siempre intervino el problema de la iluminación, así como también de las características de las tintas, los cuales hacían que varíen los resultados finales. Además, si bien el algoritmo de Lógica Difusa se convirtió en una herramienta muy versátil, y fácil de modificarla en caso de que se hubiera requerido cambiar su uso o aplicación, aun así, los resultados no son muy precisos. La etapa final se realizó controlando aisladamente unas válvulas solenoides, en un tiempo de apertura y cierre en daba paso a la liberación de cada pintura correspondiente a los colores Negro, Cian, Magenta, Amarillo y Blanco o Luminancia. Este último fue considerado como adicional por el brillo que existe en la imagen capturada.
\end{abstract}

Keywords-Lógica Difiusa, Labview, IMAQ Vision, modelos de Color RGB y HSL.

\section{INTRODUCCIÓN}

Actualmente, existen formas para la obtención de variados colores de pinturas; por ejemplo, el método tradicional de matizado. Sin embargo, este método no es preciso y es necesaria la intervención del ser humano. Es por ello que en este artículo se propone un nuevo proceso de obtención automatizado con mejor resultado y utilizando un menor tiempo. Asimismo, con la posibilidad de que pueda ser adaptada a otro tipo de proceso, haciendo más grande su campo de aplicación.

Por tal razón, se optó por emplear una de las ramas de la inteligencia artificial, la lógica difusa. Pues, los constantes avances en el campo de la inteligencia artificial, especialmente relacionado al desarrollo de sistemas expertos prácticos o sistemas de conocimientos, ha sido de considerable interés usar este nuevo campo para controlar procesos complejos. Por lo cual, una explicación razonable para usar sistemas de control inteligente puede estar relacionada a las dificultades que son comúnmente experimentadas por ingenieros de control práctico. De esta manera, un sistema basado en conocimientos busca combinar las ventajas de una computadora con algunas características de inteligencia de un humano para hacer inferencias y decisiones. Es claro que no es aconsejable intentar simplemente imitar al proceso de pensamiento de un humano, sin estudiar cuidadosamente las necesidades del problema a la mano. Por ello, una fiel duplicación no es del todo factible.

Es así, que en este artículo se describe la implementación de un sistema de control y supervisión para un proceso de pintado, utilizando una PC con Labview, una cámara web, cinco válvulas solenoides y una tarjeta de adquisición de datos. Entonces, después de capturar la imagen de un color específico mediante una webcam, se utilizó la visión artificial para procesar tal imagen y proceder a la corrección del color con el algoritmo difuso desarrollado, lo cual permitiría manipular a través de una tarjeta de adquisición de datos un conjunto de válvulas solenoides, y con ello controlar el ingreso de una determinada cantidad de color de tinta, para así obtener el resultado de un color lo más cercanos a la imagen inicialmente capturada. De esta forma, este proceso de control permitiría el ahorro de tiempo y dinero, además otorgaría precisión y versatilidad, así como un monitoreo y control desde una PC facilitando su posterior modificación en caso de ser aplicado en un nuevo proceso.

Uno de los propósitos en utilizar el modelo de color CYMK fue el trabajo de Yamada y otros, con el artículo Development of CYMK Color Display: Display by Chromogenic Effect to Control Painted Color on Paper [01], quienes controlaron digitalmente los colores de una imagen utilizando una pintura termo-sensible a los leds infrarrojos, particularmente por el profundo análisis y la manera como trabajaron con el modelo de color CYMK, a diferencia del modelo clásico de color RGB que no ofreció la información necesaria para manipular los colores que se encontraban pintados sobre un papel. De igual manera, Ramello en su artículo titulado: Comparación de métodos de detección de piel en los modelos de color $\mathrm{YCbCr}$ y HSI para reconocimiento de caras [02], utilizó esos dos modelos de color para la detección de rostros capturados como imágenes digitales. Para ello, procedió a analizar diferentes secciones de la piel y aquellas no pertenecientes al rostro. Pues, además del modelo CMYK, en tal artículo se observó la importancia del 
brillo o luminancia, así como su influencia en una imagen digital. También se notó que el procesamiento de imágenes al capturarla abrió camino a innumerables aplicaciones gracias a la diversidad de métodos con la que se cuenta para analizar una imagen digital.

\section{PRoCESAMIENTO DE IMAGEN Y CONTROL DIFUSO}

Para desarrollar el control realimentado para el prototipo de proceso industrial de pintado propuesto, se tuvo que implementar el clásico diagrama de bloques realimentado conteniendo: un controlador basado en lógica difusa, un actuador conformado por válvulas solenoides, una etapa de sensado al capturar la imagen con una webcam y posterior procesamiento con herramientas de visión artificial, y la planta industrial conformada por recipientes conteniendo tintas de variados colores.

En la figura 1, se muestra un diagrama de bloques representativo del control realimentado planteado para el desarrollo de este artículo.

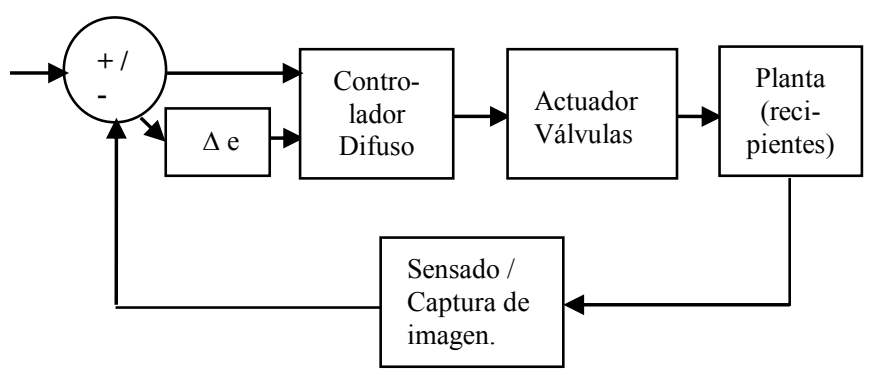

Fig. 1. Diagrama de bloques del control realimentado.

\section{A. Adquisición y almacenamiento de Imagen}

Para esta etapa fue necesario contar con el Software Labview en una version demostrativa, y sus módulos de Vision Adquisition, Vision Builder y Vision Development, los cuales permitieron realizar la captura de imágenes, y la posterior manipulación de estas. La programación se inició desarrollando una subrutina en la ventana Diagrama de Bloques, utilizando la librería "OpenCamera.VI" para configurar la webcam y la librería "ReadAttributes.VI" para cargar tal configuración. Luego, se utilizaron los instrumentos virtuales correspondientes a la grabación y captura de la imagen. Posteriormente, se utilizó la librería "Imaq.VI" que se encargó de crear una ubicación temporal para la imagen capturada, donde ahí mismo se señala el nombre del archivo y su correspondiente extensión. Asimismo, se incluyó en la programación la librería "CloseCamera.VI" encargada de cerrar la sesión de la cámara y detener por completo el proceso de captura de imagen. La figura 2 muestra la rutina de programación realizada en Labview para la configuración de la cámara, designación de la ubicación temporal y tipo de archive para la imagen capturada.

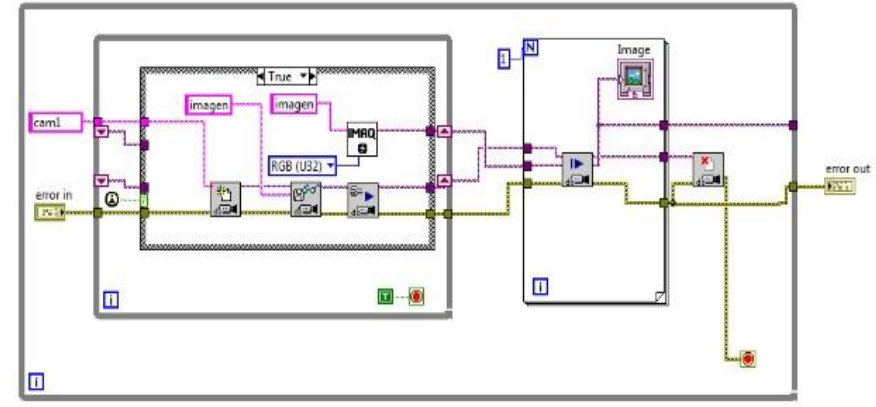

Fig. 2. Subrutina de adquisición y captura de imágenes.

Con la finalidad de simplificar y reducir los espacios de programación en la ventana Diagrama de Bloques del Labview, se optó por encapsular la programación mostrada en la figura 2. Para ello, se le designó el nombre de "Image.VI".

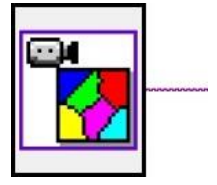

Fig. 3. Encapsulado de la subrutina de adquisición y captura de imágenes.

Complementariamente, para mejorar la precisión, se desarrolló una subrutina para seleccionar el color de interés en la imagen capturada. Para ello, se utilizaron librerías de IMAQ.VI para seleccionar la región de interés de la imagen capturada, seleccionando un área y realizando la extracción de esta. La figura 4 muestra la rutina de programación para la selección de la región de interés de la imagen capturada, incluyendo el VI de la parte correspondiente a la adquisición y almacenamiento de la imagen.

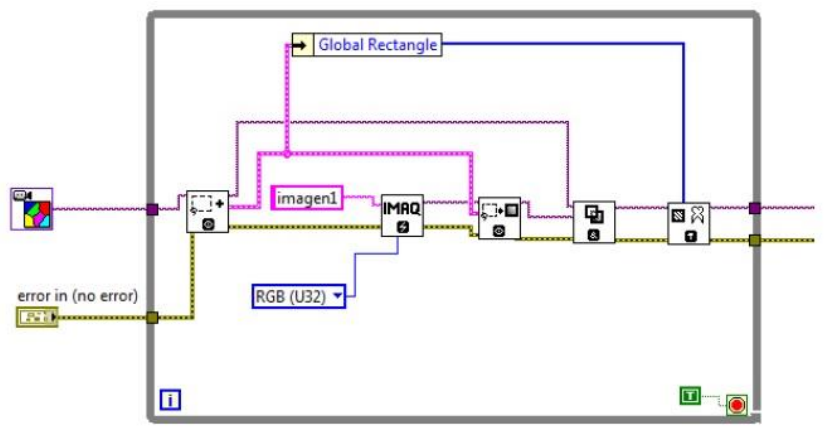

Fig. 4. Selección de la región de interés de la imagen capturada.

De igual manera, fue necesario resguardar la selección de la región de interés de la imagen capturada; para ello, se desarrolló otra subrutina de programación para almacenar tal fracción de imagen, y se señaló la carpeta de almacenamiento, además del nombre y del tipo de archivo de imagen. Ver la figura 5 . 


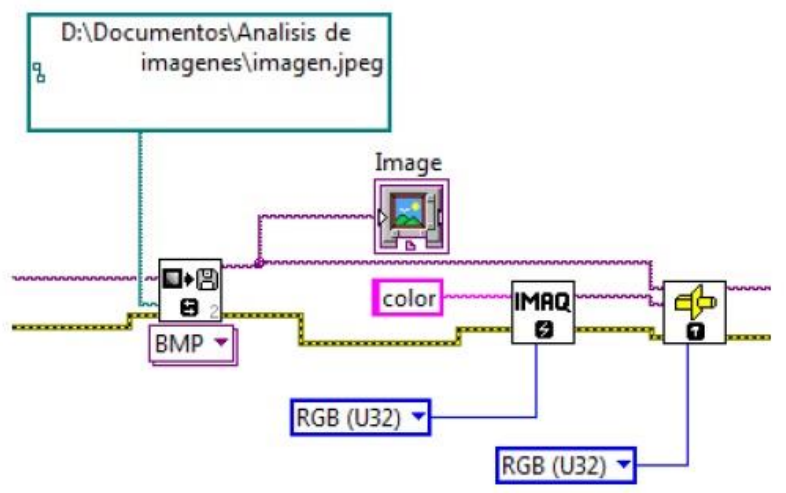

Fig. 5. Almacenamiento de la región de interés de la imagen capturada.

\section{B. Composición de Colores e Histograma}

El modelo CMYK (del acrónimo de Cyan, Magenta, Yellow and Key or Black), es un modelo de color sustractivo muy utilizado en la impresión de imágenes de colores. Además, permite representar una gama de color más amplia y tiene una mayor adaptación a los medios industriales. Por lo cual, este modelo se basa en la mezcla de pigmentos de estos cuatro colores con el fin de crear otros más.

Además de esos cuatro colores, se optó por utilizar un quinto color para representar la luminancia o luminosidad del modelo de color HSL, el cual se considera como brillo o color blanco; por ejemplo, cuando se solicita la impresión de un color claro sobre un papel del tipo bond, la intensidad de los colores CYMK se disminuye segregando menos tinta y utilizando el color blanco del papel para lograr mayor claridad. Por tal razón, y basado en la experiencia obtenida, se agregó el quinto color para el análisis de la imagen capturada.

Tal como se conoce, la mezcla ideal de colores CMY es sustractiva (puesto que la mezcla de cyan, magenta y yellow en fondo blanco da como resultado el color negro). Entonces, el modelo CMYK se basa en la absorción de la luz, y el color que presenta un objeto corresponde a la parte de la luz que incide sobre éste y que no es absorbida por el objeto. Por otro lado, siendo el color cyan opuesto al color rojo, este actúa como un filtro que absorbe dicho color (-Rojo +Verde +Azul). De igual manera ocurre con el color Magenta que es el opuesto al verde (+Rojo -Verde +Azul), y como también el amarillo que es opuesto al azul (+Rojo +Verde -Azul).

Luego de realizada la captura de la imagen de color y la toma de muestra, se procedió a determinar el histograma del modelo RGB para obtener su información de color. Esto permitió conocer la cantidad de pixels rojo, verde y azul. Seguidamente, se utilizaron los VI (instrumentos virtuales) MAX y MIN para arreglos, con el objetivo de hallar la mayor cantidad de pixels y su correspondiente tonalidad de color. Este proceso se realizó para los tres colores del modelo RGB. A continuación, la figura 6 muestra la subrutina utilizada para obtener el histograma de los colores del modelo RGB, así como también el valor de la tonalidad de cada color correspondiente a la mayor cantidad de pixels.

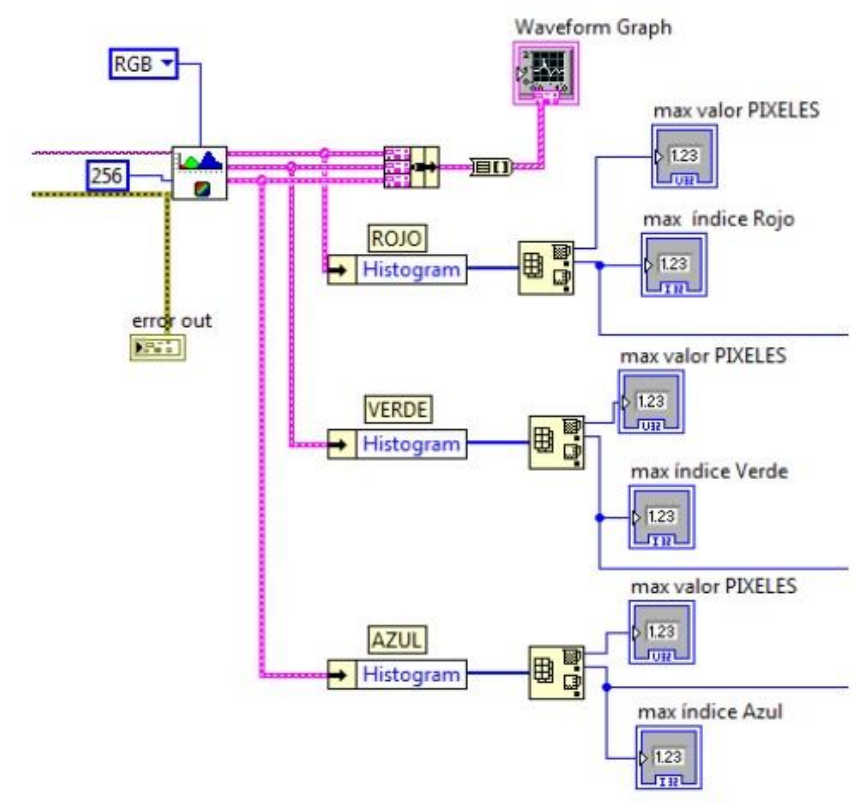

Fig. 6. Subrutina para el histograma del modelo RGB.

Una vez obtenido los valores de los colores Red, Green and Blue, se procedió a utilizar una rutina de programación en Labview para realizar la conversión al modelo CMYK más la luminancia, tal como se muestra en las siguientes ecuaciones.

$$
\begin{aligned}
& C=1-(\mathrm{R} / 255) ; \\
& \mathrm{M}=1-(\mathrm{G} / 255) ; \\
& \mathrm{Y}=1-(\mathrm{B} / 255) ; \\
& \operatorname{varK}=1 ;
\end{aligned}
$$

Y, con apoyo del condicional IF, se procedió a determinar los valores de C, M, Y y LUM para un rango de 0 al $100 \%$ para facilitar el ingreso al controlador difuso.

$$
\begin{aligned}
& \text { if }(\mathrm{C}=1 \& \mathrm{M}=1 \& \mathrm{Y}=1) \\
& \{\quad \mathrm{C}=0 ; \mathrm{M}=0 ; \mathrm{Y}=0 ; \operatorname{varK}=1 ;\} \\
& \text { else } \\
& \begin{array}{l}
\{\quad \text { if }(\mathrm{C}<=\mathrm{M} \& \mathrm{C}<=\mathrm{Y}), \operatorname{varK}=\mathrm{C} ; \\
\text { if }(\mathrm{M}<=\mathrm{C} \& \mathrm{M}<=\mathrm{Y}), \operatorname{varK}=\mathrm{M} ; \\
\text { if }(\mathrm{Y}<=\mathrm{M} \& \mathrm{Y}<=\mathrm{C}), \operatorname{varK}=\mathrm{Y} ; \\
\mathrm{C}=(\mathrm{C}-\operatorname{varK}) /(1-\operatorname{varK}) ; \\
\mathrm{M}=(\mathrm{M}-\operatorname{varK}) /(1-\operatorname{varK}) ; \\
\quad \mathrm{Y}=(\mathrm{Y}-\operatorname{varK}) /(1-\operatorname{varK}) ; \\
\} \quad \mathrm{C}=\mathrm{C} * 100 ;
\end{array}
\end{aligned}
$$




$$
\begin{aligned}
& \mathrm{M}=\mathrm{M} * 100 ; \\
& \mathrm{Y}=\mathrm{Y} * 100 ; \\
& \mathrm{LUM}=50 *(\max (\mathrm{R}, \mathrm{G}, \mathrm{B})+\min (\mathrm{R}, \mathrm{G}, \mathrm{B})) / 255
\end{aligned}
$$

De la misma manera como se procedió a encapsular una subrutina de programación realizada anteriormente en Labview, esta vez se encapsularon varias subrutinas en un solo ícono, tal como se observa en la figura 7.

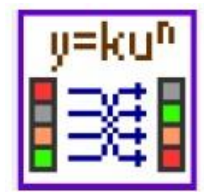

Fig. 7. Encapsulado de la programación de la captura y análisis de imágenes.

\section{Controlador difuso}

El controlador difuso utilizado en este trabajo de investigación fue diseñado de tal forma que trabaje con dos entradas. Estas son: el error y la variación del error. Y como salida el intervalo de apertura de cada una de las válvulas solenoides utilizadas. La variable de entrada "error" representó a la diferencia de la adquisición de datos de cada color de la imagen de muestra, con la adquirida al momento de realizar una prueba de color de la mezcla de tintas. Por otro lado, la entrada "variación del error" representó al porcentaje extra o faltante que pueda existir entre los errores de las pruebas de color de las siguientes mezclas. A continuación, en la tabla 1 se muestran los nombres de los conjuntos difusos utilizados con sus respectivos dominios, tanto para la variable lingüística de entrada como de salida para el controlador difuso propuesto.

\begin{tabular}{|c|c|c|}
\hline \multicolumn{3}{|c|}{ VARIABLES DE ENTRADA } \\
\hline \multirow{6}{*}{ ERROR } & Conjuntos Difusos & Dominio \\
\hline & Negativo_alto (NA) & $-50 a-25 \%$ \\
\hline & Negativo_bajo (NB) & -50 a $0 \%$ \\
\hline & Nulo (NU) & -25 a $25 \%$ \\
\hline & Positivo_bajo (PB) & 0 a $50 \%$ \\
\hline & Positivo_alto (PA) & 25 a $50 \%$ \\
\hline \multirow{6}{*}{$\begin{array}{l}\text { VARIACIÓN } \\
\text { DEL ERROR }\end{array}$} & Conjuntos Difusos & Dominio \\
\hline & Negativo_grande (NG) & $-30 a-10 \%$ \\
\hline & Negativo_pequeño (NP) & -20 a $0 \%$ \\
\hline & Cero (CE) & -10 a $10 \%$ \\
\hline & Positivo_pequeño (PP) & 0 a $20 \%$ \\
\hline & Positivo_grande (PG) & 10 a $30 \%$ \\
\hline \multicolumn{3}{|c|}{ VARIABLE DE SALIDA } \\
\hline \multirow{2}{*}{$\begin{array}{l}\text { TOTAL_IN- } \\
\text { TERVALOS_ }\end{array}$} & Conjuntos Difusos & Dominio \\
\hline & Muy_poco (MP) & 0 a 25 unid. \\
\hline
\end{tabular}

TABLA 1

Conjuntos difusos de las variables de entrada y salida.

\begin{tabular}{|c|l|c|}
\hline \multirow{2}{*}{$\begin{array}{c}\text { APERTURA } \\
\text { _VÁLVULA }\end{array}$} & Poco (PO) & 0 a 50 unid. \\
\cline { 2 - 3 } & Algo (AL) & 25 a 75 unid. \\
\cline { 2 - 3 } & Mucho (MU) & 50 a 100 unid. \\
\cline { 2 - 3 } & Demasiado (DE) & 75 a 100 unid. \\
\hline
\end{tabular}

Asimismo, el diseño de la programación del controlador difuso en el Labview, se realizó utilizando ecuaciones de la recta y condicionales para cada una de las funciones de pertenencia, de cada variable de entrada y salida empleada. Posteriormente, utilizando el entorno de programación del Labview se implementaron las reglas difusas relacionándolas con el operador AND, lo cual dio origen a 25 reglas (ver la tabla 2).

\begin{tabular}{|c|c|c|c|c|c|c|}
\hline \multirow{2}{*}{\multicolumn{2}{|c|}{ ENTRADAS }} & \multicolumn{5}{|c|}{$\operatorname{ERROR}(\%)$} \\
\hline & & \multirow{2}{*}{$\frac{\mathbf{N A}}{\mathrm{DE}}$} & \multirow{2}{*}{$\frac{\text { NB }}{\mathrm{MU}}$} & \multirow{2}{*}{$\frac{\mathbf{N U}}{\mathrm{AL}}$} & \multirow{2}{*}{$\frac{\text { PB }}{\mathrm{PO}}$} & \multirow{2}{*}{$\frac{\text { PA }}{\text { MP }}$} \\
\hline \multirow{5}{*}{ 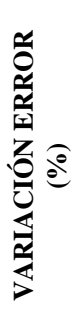 } & NG & & & & & \\
\hline & NP & MU & AL & PO & MP & MP \\
\hline & CE & AL & $\mathrm{PO}$ & MP & MP & MP \\
\hline & $\mathbf{P P}$ & PO & MP & MP & MP & MP \\
\hline & PG & MP & MP & MP & MP & MP \\
\hline
\end{tabular}

TABLA 2

Matriz de representación de las reglas difusas.

Este proceso corresponde a la operación de fuzificación y por presentar un número elevado de reglas o leyes difusas, la subrutina de programación es extensa. Por tal razón, en las figura7 y 8 se representan una parte correspondiente a la variable de entrada "error", con su respectiva salida asignada.

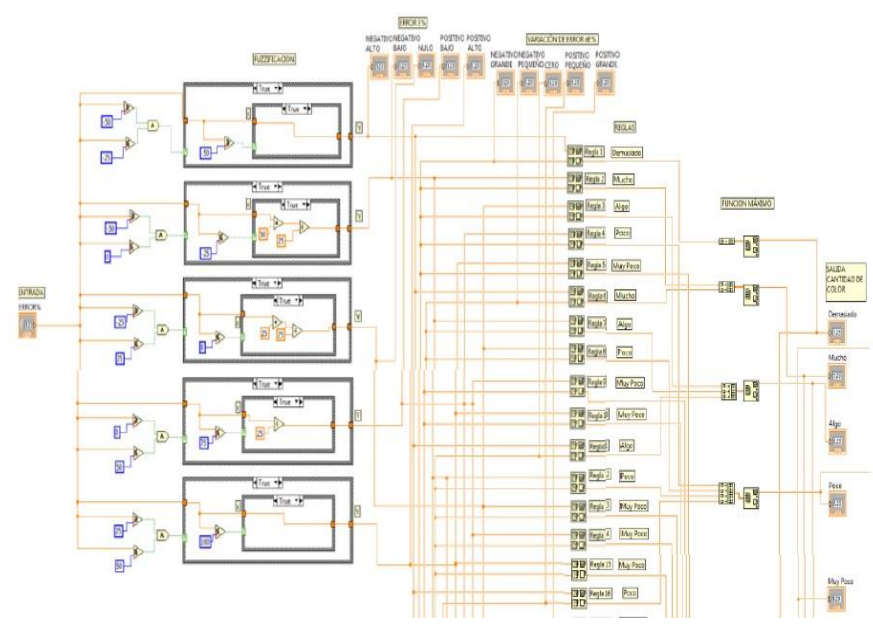

Fig. 7. Proceso de fuzificación utilizando las reglas difusas - Parte A.

$16^{\text {th }}$ LACCEI International Multi-Conference for Engineering, Education, and Technology: "Innovation in Education and 


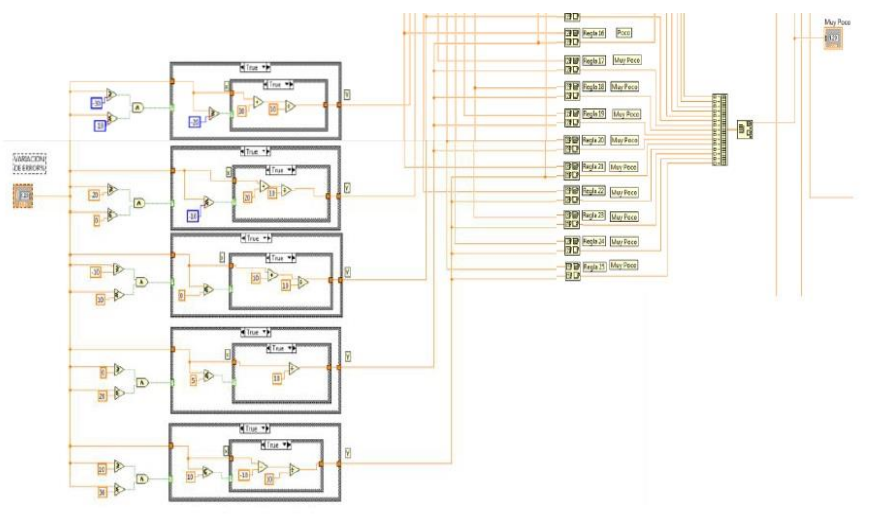

Fig. 8. Proceso de fuzificación utilizando las reglas difusas - Parte B.

Finalizada la operación de fuzificación, se continuó con la de desfusificación basado en el centro del máximo, donde la salida se determina con el valor medio de los valores cuyos conjuntos difusos o funciones de pertenencia alcanzan el máximo valor. Para ello, se representó cada recta con su respectiva pendiente, número de puntos y límites concernientes a las funciones de pertenencia de las salidas. De los datos obtenidos de la fuzificación, se procedió a separar cada ecuación de la recta que representaba a cada salida, ya sea por la función de pertenencia "MUY POCO", "POCO", "ALGO", "MUCHO" y "DEMASIADO". De esta forma, una vez indicado todo esto, se procedió a ordenar los datos correspondientes a los ejes $\mathrm{X}$ e Y de cada recta, en arreglos conformados por vectores de dimensión: 1x400.

Luego, cada valor que se tuvo como resultado de las reglas difusas, representó el límite a su correspondiente función de pertenencia de la salida. Asimismo, cada valor que se obtuvo como resultado de utilizar las reglas será el límite a su correspondiente función de pertenencia de salida. Por lo tanto, en la programación de aquel valor dado, se comparó con cada elemento que perteneció al su correspondiente eje $\mathrm{Y}$; entonces, cuando el elemento sea igual o mayor al valor originado en las reglas difusas, este es reemplazado por el número dado por la regla. De esa manera se procedió analizando y reemplazando hasta acabar con todos los elementos.

La implementación en el Labview correspondiente a la subrutina de la expresión matemática de desfuzificación y obtención del resultado final, se muestra en la siguiente figura.

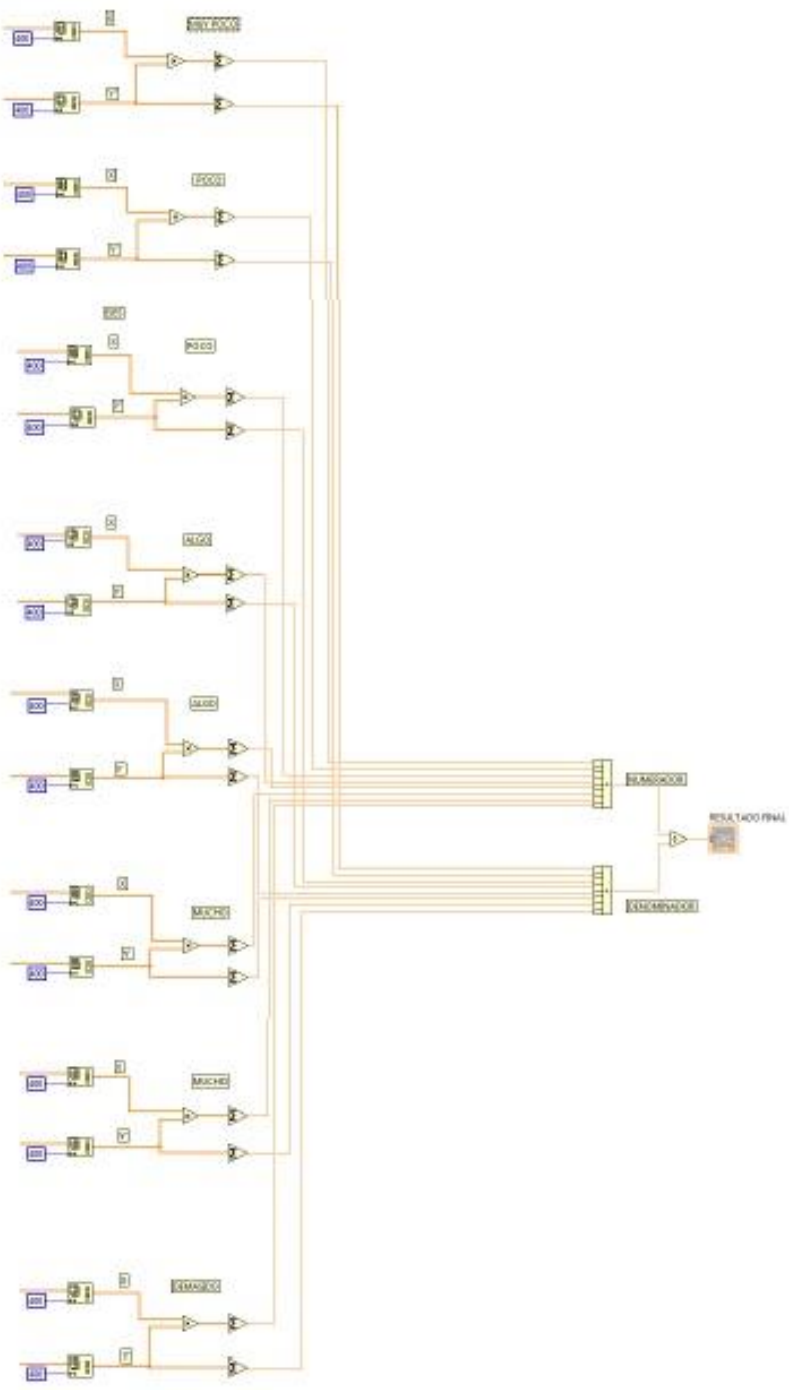

Fig. 9. Subrutina de programación para el cálculo de la fórmula de desfuzzificación.

La implementación final del controlador difuso tuvo que ser repetido 5 veces, y ser almacenado con su respectivo nombre por cada color analizado para evitar la posibilidad de que los datos sean reemplazados al utilizar una sola subrutina. Por ello, se procedió a encapsular y crear un subVI con el color respectivo, tal como se muestra en la figura 10 . Asimismo, se agregaron Vis de comparaciones con la finalidad de corregir errores en los resultados, de darse el caso de obtención de valores demasiados pequeños que podrían afectar el resultado final. Luego, a todo ello se añadió el código de programación de la captura de la imagen del resultado de la corrección del error, el cual fue ocasionado por diversos factores que influyen en la variación del color como es el caso de la luz, concentración de la pintura y el medio en que se pinta, lo cual fue necesario retroalimentarlo al controlador difuso para realizar la corrección del color en caso sea requerido. 


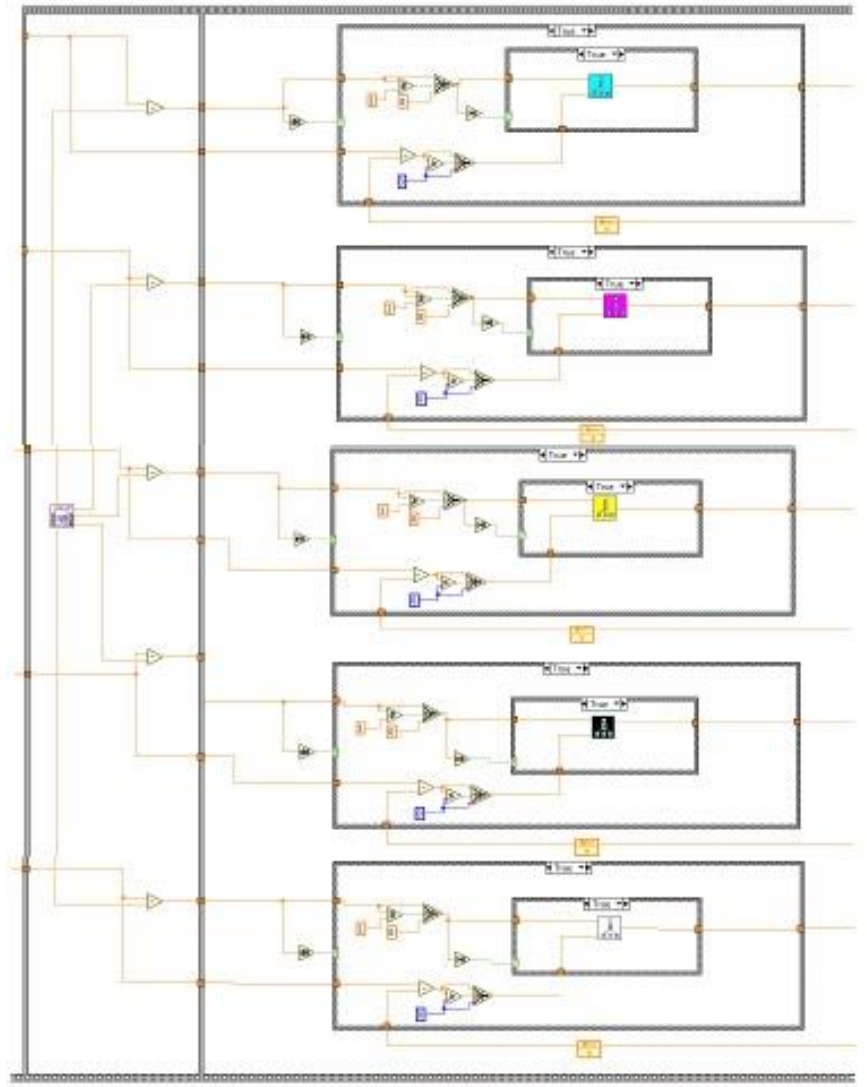

Fig. 10. Enlace de la subrutina de Lógica Difusa de cada color con la de captura de imagen.

\section{Configuración de la $D A Q$}

El modelo de tarjeta de adquisición de datos utilizada en este trabajo fue NI-USB 6501 de National Instruments, la cual se conectó a la PC via el puerto USB. Esta tarjeta cuenta con 24 líneas de entradas y/o salidas digitales, y un contador de 32 bits. A su vez, cada línea utiliza 8.5 mAmp. y 5 V. TTL/CMOS. Por lo cual, en la rutina de programación realizada en Labview se seleccionó el VI DAQ Assistant, y se eligió el Puerto 0 con sus primeras 05 líneas (line0, line1, line2, line3 y line4), y con la opción generación de señales y salida digital. Así mismo, cada línea fue conectada a la entrada de un relé de $5 \mathrm{~V}$. para controlar la apertura de las válvulas solenoides de $1 / 8{ }^{*}$ de diámetro y que funcionan con 220 VAC. Ver la figura 11. Además, tales válvulas pueden ser utilizadas tanto para líquido como para gas, y corresponden al modelo 2V025-08 con un rango de presión de trabajo de 0 a 10 bar. Luego, para facilitar la visualización de cada pintura, las válvulas se insertaron a los recipientes transparentes y a través de mangueras delgadas también transparentes, se completó el proceso con un sexto recipiente donde se realizó el vaciado final de la pintura.

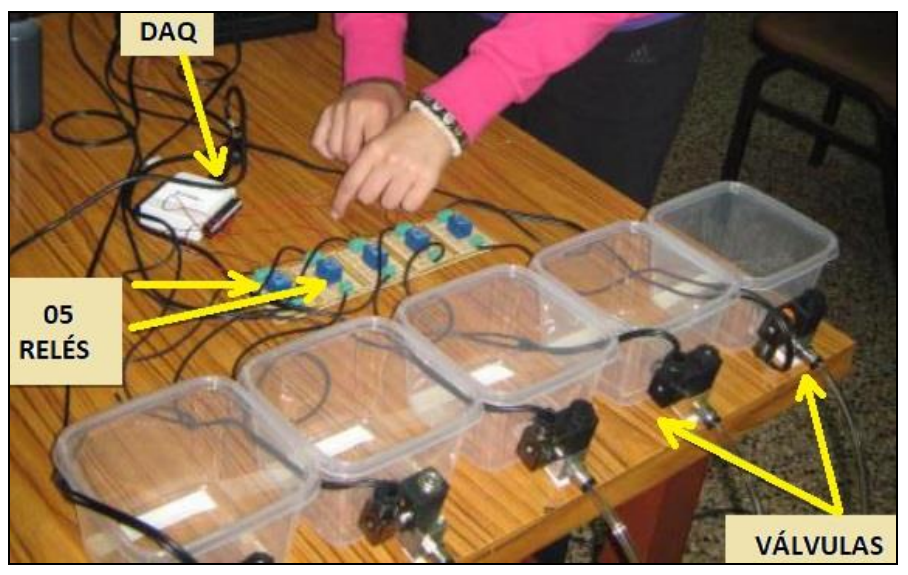

Fig. 11. Fotografía del proceso implementado: válvulas solenoides, relés y DAQ.

\section{IMPLEMENTACIÓN DEL PROCESO}

Realizada la programación en el software Labview, se procedió a la captura de las imágenes utilizando como muestras o valores de referencia retazos de cartulina de los siguientes colores: fucsia, verde, violeta, azul, celeste, amarillo, rojo y anaranjado. En la figura 12 solamente se presenta una muestra de 4 colores utilizados.
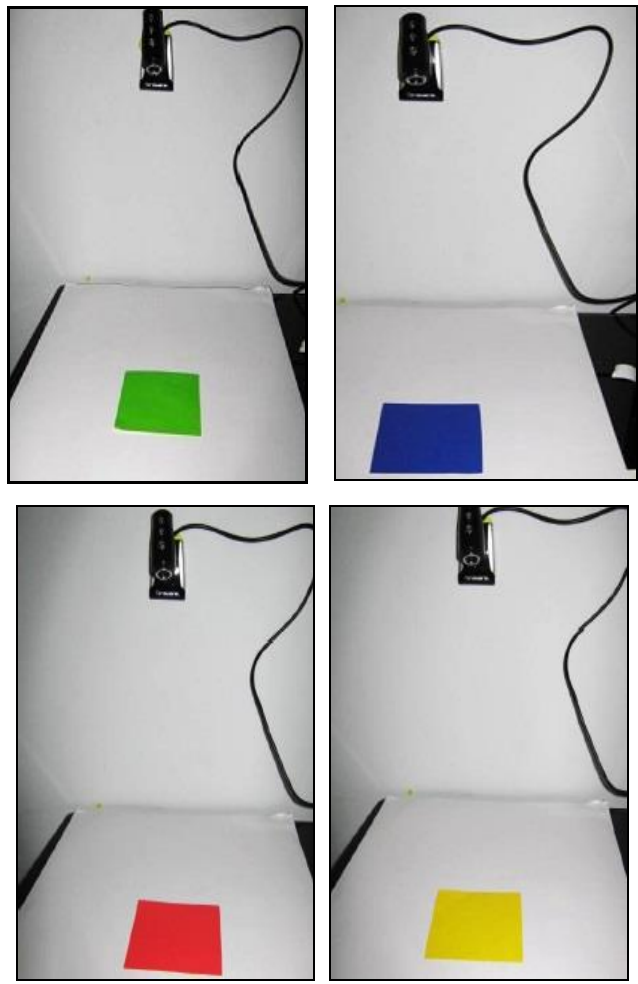

Fig. 12. Proceso de captura de imagen de muestra utilizando una Webcam.: VERDE, AZUL, ROJO y AMARILLO.

Luego, se procedió a seleccionar el área a analizar de manera manual, lo cual permitió determinar el histograma de los colores RGB y con ello se obtuvieron los datos en forma 
de arreglos, de los cuales se adquirió los valores de los niveles más altos de cada color. Estos mismos datos se utilizaron para hacer la verificación del color y comprobar que los valores rescatados al combinarlos, coincida con la muestra tomada tal como se observa en la figura 13. Por ejemplo, para el caso de esta muestra, los resultados numéricos fueron:

Máximo valor de Pixeles: 728

Máximo índice rojo:

Máximo valor de Pixeles: 1367

Máximo índice verde: 54

Máximo valor de Pixeles: 878

Máximo índice azul:

175

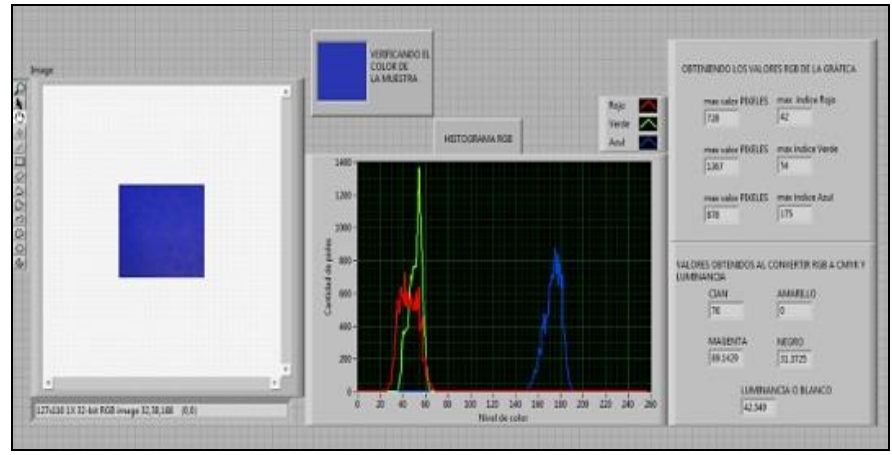

Fig. 13. Análisis de datos a partir de la imagen de una muestra.

Y, con ayuda de la programación en Labview se obtuvo la conversión al modelo CMYK (tal como también se observa en la misma figura 13), que a su vez sirvió como entrada al controlador difuso después de realizar la diferencia de colores y la variación de tal diferencia. Luego, el controlador difuso propuesto, procedió abrir las válvulas solenoides para dar paso a la salida de la pintura. Pues, la pintura utilizada correspondió a tintas de una impresora convencional que fueron ubicadas de izquierda a derecha como: AGUA, BLACK, YELLOW, MAGENTA y CYAN, tal como se observa en la figura 14. El agua fue utilizada como luminancia o color blanco. Además, como se puede observar las tintas se encuentran concentradas por corresponder a tintas de impresora, sin embargo, al utilizarse en la impresión final se utilizan pequeñas cantidades que finalmente son aclaradas al ser absorbidas por el papel.

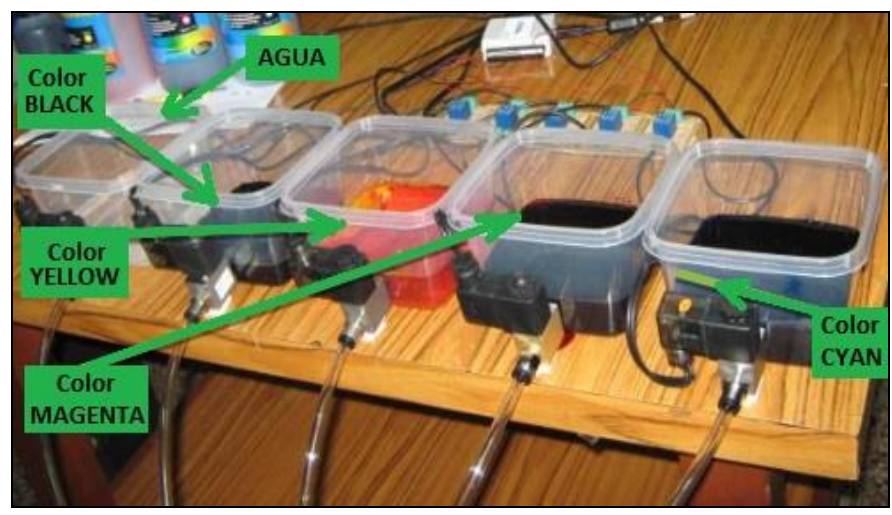

Fig. 14. Fotografía de los cinco recipientes con los colores correspondientes.

\section{RESULTADOS}

Para representar los resultados del proceso de pintado se realizaron pruebas que involucraron la mezcla de la pintura obtenida en papel, utilizando un pincel y spray para pintarlo. Luego, se procedió a la nueva captura de imagen de colores ya corregidos y que fueron pintados en papel para realizar una comparación de datos, y de esa manera se decidió si existió aún una diferencia en los valores de los colores. A continuación, se muestran dos resultados de las capturas. Ver las figuras 15 y 16.

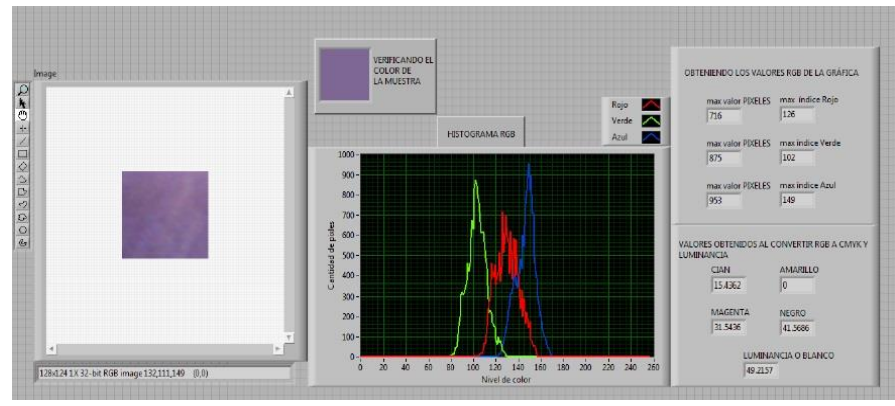

Fig. 15. Resultado de la mezcla de color violeta.

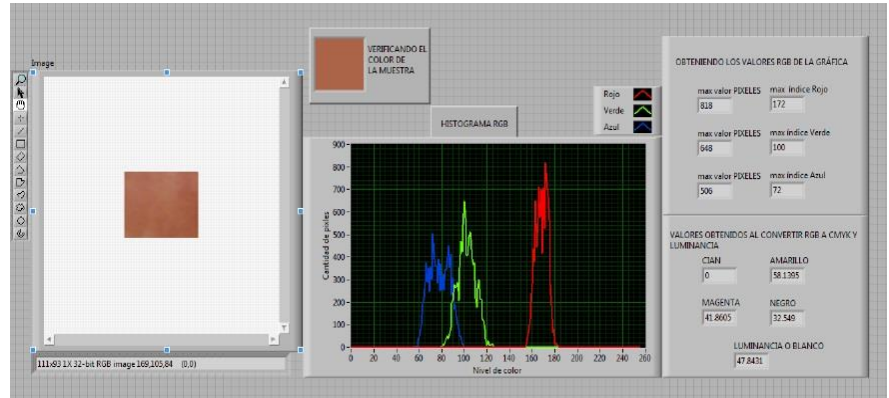

Fig. 16. Resultado de la mezcla de color rojo.

Luego, se procedió a una comparación de la composición de colores entre las muestras y sus respectivos resultados, y utilizando el error medio cuadrático se determinó el porcentaje de error entre las muestras. Ver la figura 17. 


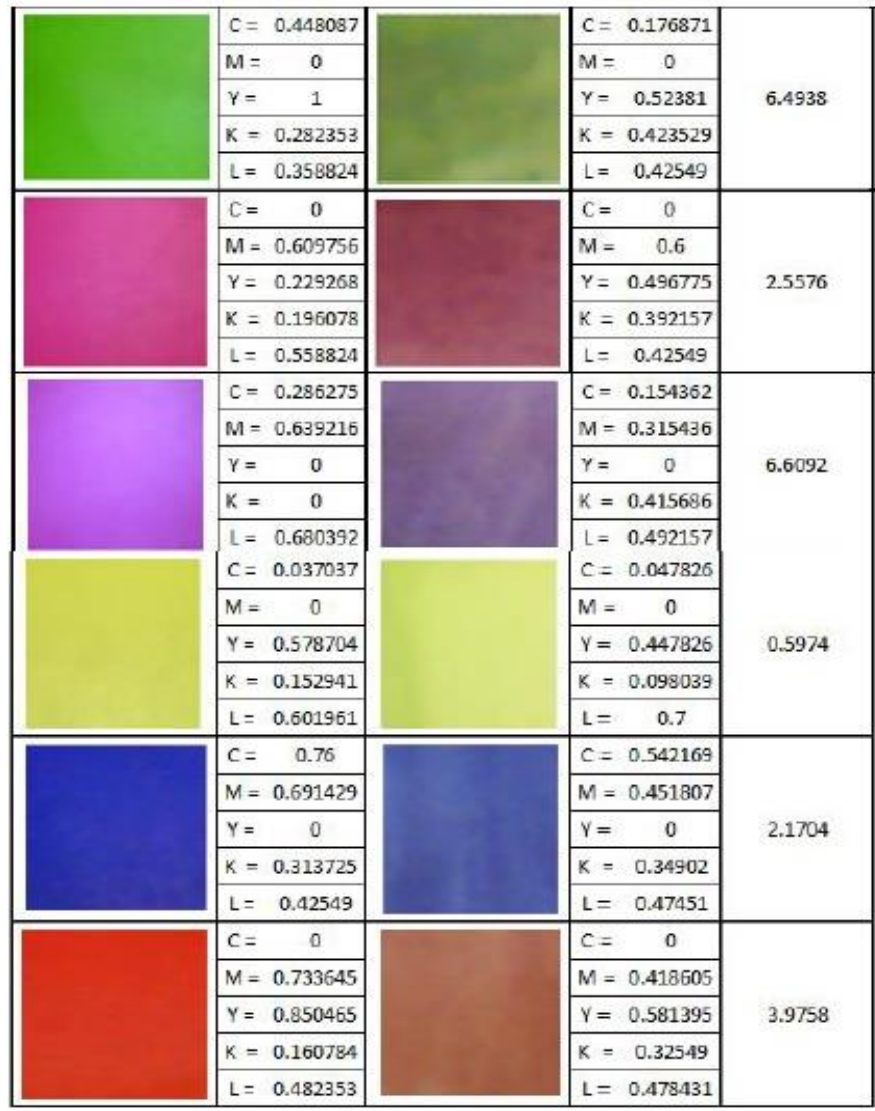

Fig. 16. Comparación de la composición de los colores entre las muestras utilizadas y sus resultados.

\section{CONCLUSIONES}

En la implementación de este proyecto se observó que la imagen de muestra capturada se vio influenciada por varios factores, entre ellos la calidad de la Webcam con que se realizó la captura. Pues, es muy importante el tipo de iluminación utilizada, sea de luz natural o artificial. Así mismo, el momento del día en que se realiza la captura. En consecuencia, se determinó que la luz artificial más perjudicial fue de color amarillenta, otorgada por lámparas incandescentes. Por tal motivo, se terminó utilizando luz artificial blanca a partir de lámparas fluorescentes.

Además, se comprobó que la lógica difusa, si bien es un método práctico y muy flexible, otorga resultados con limitaciones; es decir, las respuestas no son tan precisas como se esperaban. Esto se presentó en un caso particular con el color Negro, donde la salida de Amarillo debió ser 0 y sin embargo a través de la lógica difusa, indicó como salida un valor de 7. De esta manera, se procedió a dar paso a la salida de pintura cuando esta no era requerida. Por este motivo, es necesario realizar algunos ajustes y condiciones para otorgar mayor precisión al algoritmo de lógica difusa. Para lograr ello, intervienen las entradas de Error y Variación de Error, las cuales permiten que los resultados puedan mejorarse, realizándose más pruebas con el fin de perfeccionar el método.

La tarjeta de adquisición de datos seleccionada es el modelo más simple y de bajo costo. Sin embargo, existe una gran diferencia de precio con los demás modelos superiores a esta.

En la realización de la mezcla de pintura se pudo observar que la densidad y concentración de esta, trajo problemas para obtener un color más exacto y apropiado. Por tal motivo, fue necesario realizar más pruebas adicionando agua para acondicionar a la tinta o pintura, de esta manera lograr que la tonalidad sea tan similar al indicado por el modelo de colores CMYK. Esto se asemeja al principio de funcionamiento de la tinta de impresoras, la cual también se encuentra hecha a base de agua. Por tal motivo, se utilizó agua, como un quinto color, para aclarar los colores y lograr un brillo equivalente en la muestra de interés.

Además, se debe tener en cuenta que la pintura utilizada al ser aplicada en el papel es absorbida rápidamente y de manera dispareja, afectando también en los resultados; no se utilizó cartulina ya que tiende a absorber más tinta que el papel, se recomienda en un futuro utilizar un papel que no absorba tan rápido la tinta y pueda aplicarse la tinta en el papel de manera más pareja, como puede ser papel fotográfico. En todo caso si se utiliza otro material en lugar de la tinta de impresora, se recomienda buscar un medio más adecuado para aplicar la mezcla resultante.

En conclusión, se realizó de manera eficaz, la supervisión y control del proceso de pintado, al procesar la imagen capturada de las muestras, así como su análisis y comprobación de todos los datos obtenidos con la muestra inicial, con ayuda del algoritmo de Lógica difusa, que permitió la rectificación de los posibles errores que pudieron existir al realizar la combinación de los colores de las tintas, así como también se pudo reducir la utilización de materiales y equipos, que redujo el tiempo en el que duró el proceso

\section{REFERENCIAS}

[1] Hiroki Yamada, Kunihiro Nishimura, Tomohiro Tanikawa y Michitaka Hirose. Paper: Development of CMYk Color Display: Display by Chromogenic Effect to Control Painted Color on Paper. Universidad de Tokio. Japón. 2011.

[2] Pedro Martín Ramello. Paper: Comparación de métodos de detección de piel en modelos de color $\mathrm{YCbCr}$ y HSI para reconocimiento de caras. Universidad Nacional del Litoral. Facultad de Ingeniería y Ciencias Hídricas. Argentina.

[3] Online http://www.esacademic.com/dic.nsf/eswiki/3216

[4] Gonzalo Pajares y Jesús de la Cruz. Visión Por Computador: Imágenes Digitales y Aplicaciones. Segunda Edición. Grupo Editor Alfaomega. D.F, México. 2008.

[5] http://en.kioskea.net/contents/740-hsl-tsl-coding

[6] Clarence W. de Silva. Intelligent Control: Fuzzy Logic Applications. CRC Press, Inc. $\mathrm{N}^{\circ}$ de Edición: 0-8493-7982-2. Estados Unidos de América. 1995. 
[7] Paul P. Wang, Da Ruan, Etienne E. Kerre. Fuzzy Logic: A Spectrum of Theoretical \& Practical Issues. Editorial Springer. $N^{\circ}$ de Edición: 14349922. Berlín, Heidelberg. 2007.

[8] M. N Cirstea, A. Dinu, J.G. Khor, M. McCormick. Neural and Fuzzy Logic Control of Drives and Power Systems. Editorial Newnes. $\mathrm{N}^{\circ}$ de Edición: 07506 55585. Gran Bretaña. 2002.

[9] Rafael C. Gonzalez, Richard E. Woods. Digital image Processing. Editorial Prentice Hall Inc. Estados Unidos de América. 2002. J

[10] Jeffrey T. Spooner, Manfredi Maggiore, Raúl Ordóñez, Kevin M. Passino. Stable Adaptive Control and Estimation For Nonlinear Systems: Neural and Fuzzy Approximator Techniques. Editorial: John Wiley \& Sons, Inc. $\mathrm{N}^{\circ}$ de Edición: 0-471-41546-4. Inglaterra.2002.

[11] Kevin M. Passino, Stephen Yurkovich. Fuzzy Control. Editorial Addison-Wesley Longman, Inc. $\mathrm{N}^{\circ}$ de Edición: 0-201-18074-X. Estados Unidos de América. 1998.

[12] National Instruments Corporate Headquarters. IMAQ: IMAQ Vision Concepts Manual. Editorial: National Instruments Corporation. Estados Unidos de América. Junio 2003.

[13] National Instruments Corporate Headquarters. LabVIEW: PID and Fuzzy Logic Toolkit User Manual. Editorial: National Instruments Corporation. Estados Unidos de América. Junio 2009.

$1^{\text {th }}$ LACCEI International Multi-Conference for Engineering, Education, and Technology: "Innovation in Education and Inclusion", 19-21 July 2018, Lima, Peru. 